\title{
Persistence length and scaling properties of single-stranded DNA adsorbed on modified graphite
}

\author{
Kristian Rechendorff, Guillaume Witz, Jozef Adamcik, and Giovanni Dietler ${ }^{\mathrm{a})}$ \\ Laboratoire de Physique de la Matière Vivante, Institute of Physics of Biological Systems (IPSB), \\ Ecole Polytechnique Fédérale de Lausanne (EPFL), CH-1015 Lausanne, Switzerland
}

(Received 10 March 2009; accepted 11 August 2009; published online 2 September 2009; publisher error corrected xx September 2009)

\begin{abstract}
We have characterized the polymer physics of single-stranded DNA (ssDNA) using atomic force microscopy. The persistence length $\ell_{p}$ of circular ssDNA adsorbed on a modified graphite surface was determined independently of secondary structure. At a very low ionic strength we obtained $\ell_{p}=9.1 \mathrm{~nm}$ from the bond correlation function. Increasing the salt concentration lead to a decrease in $\ell_{p}$; at $1 \mathrm{mM} \mathrm{NaCl}$ we found $\ell_{p}=6.7 \mathrm{~nm}$, while at $10 \mathrm{mM} \mathrm{NaCl}$ a value $\ell_{p}=4.6 \mathrm{~nm}$ was obtained. The persistence length was also extracted from the root-mean-square end-to-end distance and the end-to-end distance distribution function. Finally, we have investigated the scaling behavior using the two latter quantities, and found that on long length scales ssDNA behaves as a two-dimensional self-avoiding walk. (C) 2009 American Institute of Physics. [doi:10.1063/1.3216111]
\end{abstract}

\section{INTRODUCTION}

Single-stranded DNA (ssDNA) constitutes the genetic material in certain viruses, and it is an important intermediate in many key biochemical processes, such as replication and transcription. In its interactions with other macromolecules, e.g., proteins, the flexibility plays a significant role, ${ }^{1}$ and a good understanding of the statistical and mechanical properties of ssDNA is therefore highly desirable. However, elucidating the polymer physics of ssDNA has proved difficult due to its tendency to form intrastrand base pairs, ${ }^{2,3}$ which in turn lead to a compact polymer conformation. This makes the comparison between experimental results and simple polymer theories difficult, as the latter usually assumes that the polymer can be described in terms of a small number of parameters, such as the persistence length or the scaling exponents. ${ }^{4}$ The fact that DNA molecules (single-as well as double-stranded) are charged in solution adds to the difficulties of both the theoretical description and the interpretation of the experimental findings. It is well-established that the flexibility of a polyelectrolyte increases with increasing ionic strength, because the higher salt concentration allows for a more effective screening of the charges on the polymer. The flexibility is characterized by the persistence length $\ell_{p}$, which is the decay length of the orientational bond correlations. If electrostatic interactions are present, the persistence length is usually divided into two parts; an intrinsic part which is independent of salt concentration and an electrostatic part. The precise dependence of the electrostatic persistence length on the ionic strength is a complex, and still debated, issue. ${ }^{5}$ For ssDNA the decrease in the electrostatic persistence length as a function of salt leads to an additional increase in the propensity to form intrastrand base pairs. This effect potentially leads to biased values of persistence length when $\ell_{p}$ is extracted from experimental data: If $\ell_{p}$ is ob-

\footnotetext{
${ }^{a)}$ Electronic mail: giovanni.dietler@epfl.ch.
}

tained from the global properties of the chain (radius-ofgyration), as typically done in scattering experiments, ${ }^{4}$ it will be found using the relation $R_{G}^{2} \propto \ell_{p} L$ in the limit of a long chain. However, with base pairing present, one can no longer assume such a relation to be reliable, because the chain will be shorter than its nominal length $L$, and because the stiffness of the chain cannot be described with a single value of $\ell_{p}$. Also, if the radius-of-gyration is found only for a single chain length it is impossible to know whether the ideal chain relation $R_{G}^{2} \propto \ell_{p} L$ is valid, or if self-avoidance cannot be neglected, and one should use a relation of the type $R_{G}^{2} \propto L^{\nu}$. In order to experimentally determine the persistence length of ssDNA, researchers have tried to circumvent the problem of base pairing by using specific chemical agents that prevent base pairing, e.g., glyoxal, ${ }^{3}$ or by using synthetic polynucleotide sequences where base pairing is less likely, e.g., poly(dT) (Refs. 6-10) or poly(U). ${ }^{11,12}$ These studies have shown that ssDNA is a very flexible polymer, as was already expected from other experiments, ${ }^{13,14}$ with a persistence length in the order of a few nanometers. However, it cannot be excluded that chemical modifications induce changes in the molecular structure, and, as a result, the values of $\ell_{p}$ may be affected. To further clarify the situation, additional studies using other techniques are needed. An alternative experimental route is to study ssDNA adsorbed on a surface using atomic force microscopy (AFM) or fluorescence. Obviously, adsorbing the polyelectrolyte on a surface can lead to changes in its conformation that are difficult to quantify, and, consequently, the value of $\ell_{p}$ may differ from the results of three-dimensional (3D) measurements. ${ }^{15}$ Nevertheless, studies in which double-stranded DNA (dsDNA) was adsorbed onto microscopically flat substrates, and subsequently imaged with AFM, have yielded significant insight into the polymer physics of this molecule, ${ }^{16-20}$ as have studies of dsDNA adsorbed onto lipid bilayers and characterized by fluorescence. ${ }^{21,22}$ Due to its high resolution combined with 
the ability to scan large areas, AFM allows for a precise quantification of the polymer physics on a wide range of length scales. As a consequence the persistence length can be determined not only from the global properties (e.g., the radius of gyration), but also from the short-scale structure as encoded in the bond correlation function for nearby segments. Finally, AFM imaging avoids the problem of an unknown molecular length; all quantities (bond correlations, end-to-end distance, etc.) are measured directly as a function of contour length. In contrast to fluorescence no chemical labeling of the molecules is necessary. For ssDNA the main advantage of a direct visualization of the molecules is that secondary structure formation is readily visible, allowing for a determination of the persistence length from purely singlestranded regions. Previous AFM investigations have shown that ssDNA could be imaged under certain experimental conditions; ${ }^{23-26}$ however, these studies did not address the polymer physics.

In this paper, we study the polymer physics of circular ssDNA adsorbed onto a modified graphite surface. Our aim is to find values of the persistence length at different salt concentrations, independently of secondary structure, using the decay of the bond correlation function, the internal endto-end distances, and the distribution of end-to-end distances. From the decay of the bond correlations we find $\ell_{p}=9.1 \mathrm{~nm}$ at very low salt and 6.7 and $4.6 \mathrm{~nm}$ at 1 and $10 \mathrm{mM} \mathrm{NaCl}$, respectively. Values obtained from the internal end-to-end distance and the distribution function correspond quite well with this. In addition, we characterize the scaling behavior, and find that ssDNA on the surface can be described as a self-avoiding walk (SAW) in two-dimensional (2D).

\section{EXPERIMENTAL SECTION}

The highly oriented pyrolytic graphite (HOPG) surface (ZYA quality from MikroMasch, Tallin, Estonia) was cleaved with Scotch tape, and subsequently it was modified using a compound consisting of a hydrocarbon-peptide chain terminated with an amine group: $\left(\mathrm{CH}_{2}\right)_{n}\left(\mathrm{NC}(\mathrm{Gly}) \mathrm{H}_{2}(\mathrm{CO})\right)_{m} \mathrm{NH}_{2}$, (Gly is glycine) from Nanotuning, Chernogolovka, Russia. $^{26-28}$ These molecules form a thin $(\sim 0.7 \mathrm{~nm})$ monolayer, with the $\mathrm{NH}_{2}$-group exposed to the liquid. The resulting modification of the surface is similar to the well-known aminopropyltriethoxysilane (APTES) functionalization of mica; ${ }^{29,30}$ in an aqueous solution the amine group becomes protonized creating a positively charged surface, onto which DNA can adsorb. Circular ssDNA, Virion $\phi$ x174 (5386

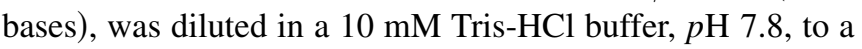
final concentration of $10 \mu \mathrm{g} / \mathrm{ml}$. For the adsorption of ssDNA, $1 \mu$ l DNA solution was dissolved in $30 \mu$ l of ultrapure water, containing either 0,1 , or $10 \mathrm{mM} \mathrm{NaCl}$, and allowed to adsorb to the surface for $7 \mathrm{~min}$. The surface was then gently blow-dried with air, and the sample was imaged by AFM in intermittent mode under ambient conditions at a scan frequency of $1 \mathrm{~Hz}$. Silicon nitride cantilevers (MicroMasch) with a typical tip radius below $10 \mathrm{~nm}$ were used. Images were flattened with the AFM software, and no further image processing was done.
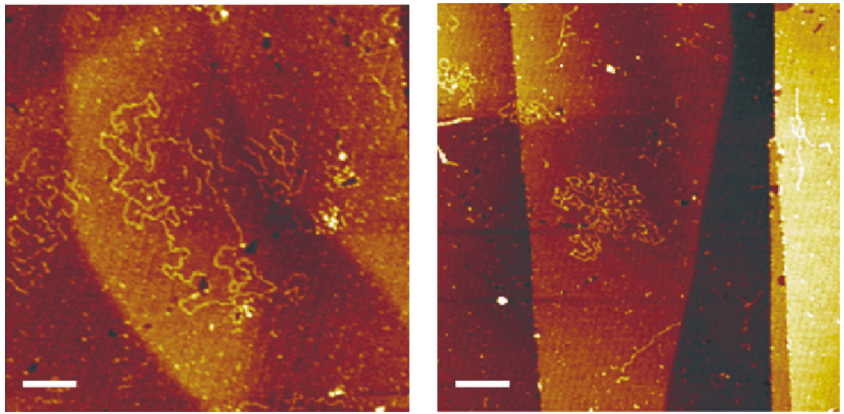

FIG. 1. Representative AFM images of circular ssDNA adsorbed on modified HOPG. Left image: $0 \mathrm{M} \mathrm{NaCl}$ and right image: $10 \mathrm{mM} \mathrm{NaCl}$. Bar size is $100 \mathrm{~nm}$.

\section{RESULTS}

\section{A. AFM images}

The left image in Fig. 1 shows ssDNA adsorbed on modified HOPG in the case of no added salt. Compared to the cases of ssDNA adsorption on mica in the presence of $\mathrm{Mg}^{2+}$ ions, or on APTES functionalized mica, ${ }^{26}$ the molecules adsorbed on modified HOPG at low salt have an open conformation without secondary structure. In addition, we note that the molecules exhibit very few crossings. From similar images, the contour of 52 molecules was traced using the software ELLIPSE. ${ }^{31}$ The average length of the molecules is found to be $1975 \mathrm{~nm}$, which equals a length per base of $0.37 \mathrm{~nm}$ in agreement with a previous investigation where, depending on sequence, $0.32-0.52 \mathrm{~nm}$ was found. ${ }^{6}$ Values for the height and width of the adsorbed molecules are similar to the reported values from other AFM studies, 0.3 and $7 \mathrm{~nm}$, respectively. ${ }^{25,26}$ The right image in Fig. 1 shows a molecule adsorbed from a $10 \mathrm{mM} \mathrm{NaCl}$ solution. The enhanced screening of the backbone charges lead to a significantly more compact polymer conformation, and, as a result, we could only partially trace the coordinates of the molecules, hence no estimate of the total length was obtained. The images pertaining to a $1 \mathrm{mM} \mathrm{NaCl}$ solution exhibit characteristics which lie in between the 0 and $10 \mathrm{mM}$ cases.

\section{B. Bond correlations}

A fundamental quantity characterizing the conformation of a polymer is the orientational correlation among the segments. In models with a local bending rigidity, such as the wormlike chain (WLC) model, the persistence length can be found from the exponential decay of the correlations between the unit tangent vectors $\vec{u}$ belonging to different segments separated by the distance $s$ along the chain: $\langle\vec{u}(l) \cdot \vec{u}(l$ $+s)\rangle=\langle\cos \theta(s)\rangle=\exp \left(-s / 2 \ell_{p}\right)$. For an ideal, linear polymer this decay provides a valid description for all values of $s$; for large separations (compared to $\ell_{p}$ ) the chain simply corresponds to a Gaussian chain. If the conformation is influenced by other effects besides stiffness, for example, self-avoidance and/or a topology other than linear, the exponential decay can only be expected to provide a good approximation for small segment separations. For a SAW power-law corrections will appear on longer length scales, ${ }^{32}$ and details regarding the correlations in a circular molecule can be found 


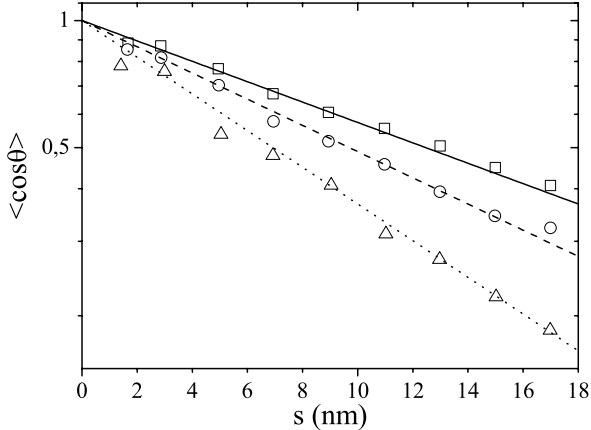

FIG. 2. The decay of $\langle\cos \theta(s)\rangle$ as a function of segment separation $s$ for the three cases 0,1 , and $10 \mathrm{mM} \mathrm{NaCl}$. Fitting with $\exp \left(-s / 2 \ell_{p}\right)$ gives $\ell_{p}$ $=9.1 \pm 0.3 \mathrm{~nm}$ (full line), $6.7 \pm 0.2 \mathrm{~nm}$ (dashed line), and $4.6 \pm 0.3 \mathrm{~nm}$ (dotted line), respectively.

in Refs. 33 and 34. In the decay we employ $2 \ell_{p}$ as the characteristic decay length. This means that, provided the molecules are in an equilibrated 2D conformations, we get information about the 3D persistence length. ${ }^{35}$ In Fig. 2 is shown $\langle\cos \theta(s)\rangle$ for the three different salt concentrations. At low salt, a fit to the exponential decay results in $\ell_{p}$ $=9.1 \pm 0.3 \mathrm{~nm}$. Increasing the salt concentration we observe a clear decrease in $\ell_{p}$. At $1 \mathrm{mM} \mathrm{NaCl}$ we find $\ell_{p}$ $=6.7 \pm 0.2 \mathrm{~nm}$, and at $10 \mathrm{mM} \mathrm{NaCl} \ell_{p}=4.6 \pm 0.3 \mathrm{~nm}$.

\section{Internal end-to-end distance}

The conformation of a polymer is often described using the end-to-end distance. This quantity is closely related to the bond correlations, and one can obtain the same information concerning the persistence length and scaling exponents from either of the two ${ }^{36}$ For a circular molecule it is useful to study the internal end-to-end distances $\left\langle\xi^{2}(s)\right\rangle^{1 / 2}$, which measure the segment separations within the molecule as a function of contour length $s$. In Fig. 3, we show the $\left\langle\xi^{2}(s)\right\rangle^{1 / 2}$ data in the low salt case. Several regimes exist depending on the contour length, and evidently the curve is symmetric around $L / 2$. For small to moderate values of $s$ the effect of circularity can be ignored, and for the very smallest values of $s$ a linear scaling is found, $\left\langle\xi^{2}(s)\right\rangle^{1 / 2} \simeq s$. In this regime the molecules can be viewed as rigid rods. Increasing $s$, leads

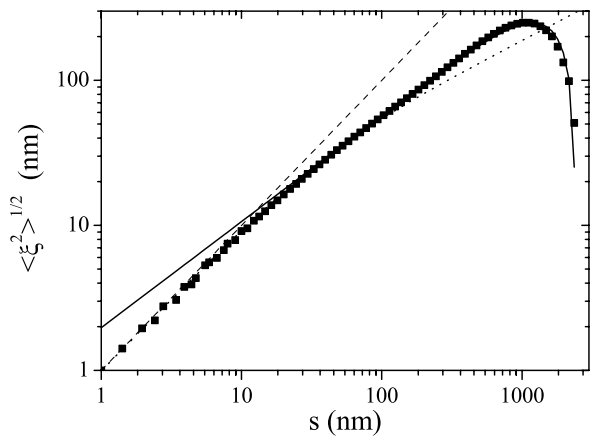

FIG. 3. The internal mean-square end-to-end distance as a function of contour length at $0 \mathrm{M} \mathrm{NaCl}$. For small $s$ the data follows a line with slope one (dashed line) corresponding to rigid rod behavior of the molecules. The WLC model [Eq. (1)] (dotted curve), gives a good description of the data with $\ell_{p} \simeq 9 \mathrm{~nm}$ for (see text). For large contour lengths fitting with Eq. (2) gives values $\nu=0.73 \pm 0.01$ and $L=2315 \pm 15 \mathrm{~nm}$ (full curve). first to the semiflexible, and then the flexible regime. We compare the data to the WLC model in order to find the persistence length. In 2D the WLC expression for the endto-end distance is given by

$$
\left\langle\xi^{2}(s)\right\rangle=4 \ell_{p} s\left[1-\frac{2 \ell_{p}}{s}\left(1-\exp \left(-\frac{s}{2 \ell_{p}}\right)\right)\right] .
$$

We fit the data for the internal end-to-end distances with Eq. (1), as shown in Fig. 3, and find values of $\ell_{p}$ between 7 and $12 \mathrm{~nm}$ depending on the range of $s$ used in the fit (values of $s$ between 30 and $300 \mathrm{~nm}$ all give reasonable fit). To avoid the question of the proper range of fitting, we find the value of $s$ at which Eq. (1) with $s=\ell_{p}$, that is $\left\langle\xi^{2}(s)\right\rangle=(0.923 s)^{2}$, crosses the data. This crossing gives $\ell_{p}=9.0 \pm 0.5 \mathrm{~nm}$ (the uncertainty is an estimate), which confirms the finding from the bond correlation. From Fig. 1, it is clear that for segment separations more than about $200 \mathrm{~nm}$, the WLC model underestimates the measured end-to-end distance. The reason for this is self-avoidance, which makes the polymer swell compared to the ideal chain. Self-avoidance is not taken into account in Eq. (1); in the limit $s \gg \ell_{p}$ the scaling is $\left\langle\xi^{2}(s)\right\rangle^{1 / 2} \sim s^{1 / 2}$, corresponding to a Gaussian chain. For a linear molecule, a power-law fit $\left(\left\langle\xi^{2}(s)\right\rangle^{1 / 2} \sim s^{\nu}\right)$ would be sufficient to determine the critical exponent $\nu$, however, for a ring polymer, both self-avoidance and circularity are important at these scales. To take these two effects into account, we fit with the approximate formula, ${ }^{37}$

$$
\left\langle\xi^{2}(s)\right\rangle \propto \frac{s^{2 \nu}(L-s)^{2 \nu}}{s^{2 \nu}+(L-s)^{2 \nu}} .
$$

Experimental studies of this function are scarce, nevertheless, a recent work showed that it provides a good description of circular dsDNA. ${ }^{33}$ A fit for values of $200 \mathrm{~nm}<s$ $<L-200 \mathrm{~nm}$ results in $\nu=0.73 \pm 0.01$, and a total length of the molecules $L=2315 \pm 15 \mathrm{~nm}$, the latter being larger than the direct result from the AFM images, because the large total lengths have more weight in this fit compared to the direct average. The value of the critical exponent is very close to the result for a 2D SAW, where $\nu=0.75 .{ }^{4}$ From AFM studies of linear dsDNA adsorbed on modified HOPG ${ }^{38}$ and on spermine ${ }^{18}$ similar results for the critical exponent were obtained. In addition, these studies found a scaling regime for $\left\langle\xi^{2}(s)\right\rangle^{1 / 2}$ with Gaussian behavior $(\nu=0.5)$ between the rigid rod and the SAW regimes. The absence of this Gaussian regime in the present case is most likely due to the dimensionality and the topology of the adsorbed DNA. Collisions between two strands are more likely in 2D, and circularity requires that the polymer strand returns to its origin. Both effects increase the self-avoiding interaction on short distances. AFM experiments with circular dsDNA on $\mathrm{Mg}^{2+}$-mica (Ref. 33) and theoretical investigation of semiflexible ring polymers ${ }^{39}$ confirm this conjecture.

\section{End-to-end distributions}

Single-molecule techniques, such as AFM, allow investigation of the distribution of end-to-end distances, $G(\xi)$. The persistence length and the critical exponents can be extracted from $G(\xi)$ in the semiflexible and flexible limits, respec- 


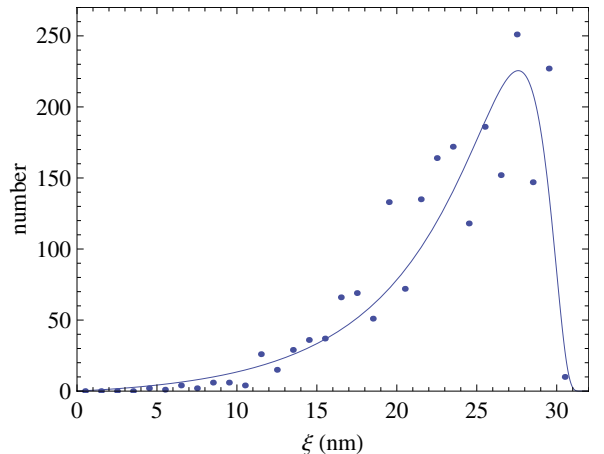

FIG. 4. Experimental data for the radial end-to-end distance distribution for a contour length $s=32 \mathrm{~nm}$ at $0 \mathrm{M} \mathrm{NaCl}$ compared to the theoretical distribution [Eq. (5)] from Ref. 40. Fitting gives $\ell_{p}=6.5 \mathrm{~nm}$.

tively. Hence, the distribution is an important complement to the more frequently studied quantities such as the end-to-end distance or the bond correlation function. We consider the 2D radial distribution function $f(\xi)=2 \pi \xi G(\xi)$ of internal end-to-end distances. For contour lengths below approximately $200 \mathrm{~nm}$ the polymer behaves as a semiflexible chain, and the WLC model provides a good description of the data, see Fig. 3. The appropriate distribution in this regime is the one for a WLC. Above a contour length of $200 \mathrm{~nm}$ the effect of self-avoidance becomes important, and the form of the distribution is that of a SAW. This distribution is distinctively different from the one at short scales (where the persistence length enters as a parameter), and functions belonging to different values of the contour length should collapse onto a single graph provided the correct scaling exponent is used.

We first consider the semiflexible regime, that is, $s \simeq \ell_{p}$. The exact form of the distribution of end-to-end distances in a WLC chain is presently unknown, nevertheless, several approximate results exist. ${ }^{40-43}$ For a polyelectrolyte the problem is further complicated by electrostatic effects. ${ }^{44}$ Here, we restricts ourselves to a comparison of the data for contour lengths below $100 \mathrm{~nm}$ to the theory developed in Ref. 40. In a later publication we will pursue the subject of end-to-end distributions of a semiflexible polymer further. The result of Ref. 40 is based on a perturbation expansion of the tangent field using a rigid rod as a reference, and it is expected to be valid only for $s<\ell_{p}$. It turns out, however, that the result remains useful also for much higher $s$-values. In Fig. 4 we show the data for the end-to-end distribution for $s=32 \mathrm{~nm}$. Fitting our data with the theory [Eq. (5) in Ref. 40], we find $\ell_{p} \simeq 6.5 \mathrm{~nm}$, lower than the value from the bond correlations and from the end-to-end distance. ${ }^{45}$ The reason for this discrepancy among the values for $\ell_{p}$ is most likely the low salt content of the solution, which makes the result for the distribution a less good approximation. Increasing the salt concentration we find lower values of $\ell_{p}$ in good accordance with the values found from the bond correlations and the end-toend distances. The values are summarized in Table I.

In the limit $s \gg l_{p}$, self-avoidance becomes important and the distribution of $\xi$ has the form ${ }^{46,47}$
TABLE I. The persistence length for ssDNA at different salt concentrations obtained from the bond correlations $\langle\cos (\theta)\rangle$, the end-to-end distance $\left\langle\xi^{2}\right\rangle$, and the radial end-to-end distribution $f(\xi)$. The Debye screening length is in parentheses. All lengths are in nanometers.

\begin{tabular}{lccc}
\hline \hline & $0 \mathrm{M}(>100 \mathrm{~nm})$ & $1 \mathrm{mM}(9.6 \mathrm{~nm})$ & $10 \mathrm{mM}(3 \mathrm{~nm})$ \\
\hline$\langle\cos (\theta)\rangle$ & $9.1 \pm 0.3$ & $6.7 \pm 0.2$ & $4.6 \pm 0.3$ \\
$\left\langle\xi^{2}\right\rangle$ & $9.0 \pm 0.5$ & $5.5 \pm 0.5$ & $4.4 \pm 0.5$ \\
$f(\xi)$ & $\sim 6.5$ & $\sim 5.0$ & $\sim 4.4$ \\
\hline \hline
\end{tabular}

$$
f(z)=\left\{\begin{array}{cc}
\mathcal{A} z^{D-1+\kappa} \exp \left(-\mathcal{B} z^{\delta}\right) & \text { if } z \gg 1 \\
\mathcal{C} z^{D-1+\theta} & \text { if } z \ll 1,
\end{array}\right.
$$

where $z=\xi /\left\langle\xi^{2}\right\rangle^{1 / 2}$, and $\mathcal{A}, \mathcal{B}$, and $\mathcal{C}$ are constants. Apart from depending on the dimensionality $D$ the critical exponents $\kappa$, $\delta$, and $\theta$ depend on the specific points between which the correlation is considered (two end-points, one end-point and one interior point, and two interior points). As we deal with circular molecules, only two interior points are relevant. In $2 \mathrm{D}$, the exact value of the exponent $\theta$ was found analytically, ${ }^{48}$ and for two interior point $\theta=19 / 12 \simeq 1.583$. If the scaling relation $\delta=1 /(1-\nu)$ (Ref. 46) is assumed to hold for circular molecules, $\delta=4$ for a 2D SAW. However, studies using simulations and exact enumeration have pointed to a value close to 4.6 instead (see Table I in Ref. 47). Different values were also obtained for the other exponents (see Ref. 47 for a summary of the studies). Although the distribution has been subject to extensive theoretical investigations, the experimental confirmations of Eq. (3) are very few. ${ }^{17}$ In Fig. 5 is shown the collapse of the distributions with contour lengths of 210 and $315 \mathrm{~nm}$ when these are rescaled using $\nu$ $=0.73$. We observe a good data collapse, confirming the value of the critical exponent $\nu$ found from the end-to-end distances. Also shown are the two limits of Eq. (3); a power law with exponent $\theta=1.583$ follows the data well in the regime $z \ll 1$, whereas $\exp \left(-b z^{\delta}\right)$ with $\delta=4.6$ gives a good description of the data in the limit $z \gg 1$. It should be noted, that

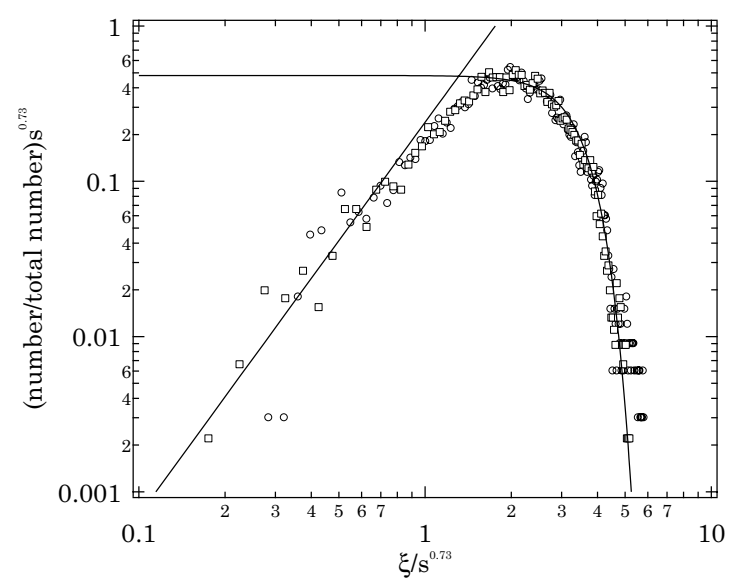

FIG. 5. The distribution of end-to-end distances for long contour lengths at $0 \mathrm{M} \mathrm{NaCl}$. $s=210 \mathrm{~nm}$ (squares) and $s=315 \mathrm{~nm}$ (circles), rescaled using $\nu$ $=0.73$ to obtain data collapse. Comparison with Eq. (3) was done; for small $z$-values a power law with $D-1+\theta=2.583$ is shown, while for large $z$-values and exponential with $\delta=4.6$ is drawn. 
from the data it is difficult to determine whether $\delta=4$ or $\delta$ $=4.6$, as both these values will result in a reasonable agreement between the data and Eq. (3).

\section{DISCUSSION}

A direct visualization of ssDNA with AFM is very useful for studying the conformation of the molecule. Accompanying the decrease in the chain stiffness with salt, there is an increase in base pair formation, and direct imaging allows one to observe the onset of base pair formation and to estimate the persistence length from purely single-stranded regions. However, a main critic of AFM studies is that the molecules are adsorbed on a surface.

If the ssDNA is in a purely $2 \mathrm{D}$ conformation on the surface, one can use the results of $2 \mathrm{D}$ polymer theory to interpret the data. From an experimental perspective the scaling exponent $\nu$ provides the easiest way of determine the dimensionality of the polymer, although this is only valid on long scales. One is therefore unable to rule out a potentially different structure on very short scales using the critical exponents. Several AFM studies ${ }^{16,17,20}$ on dsDNA have identified two extreme cases: The situation where the polymersurface interaction allows for sufficient rearrangement so that the polymer may be considered $2 \mathrm{D}$, and the case where the interaction leads to a quick adsorption which leaves the molecules in a projection of their 3D state. In addition, partially relaxed conformations may also appear. For dsDNA adsorbed on a mica surface with magnesium added in solution to work as a binding agent, the conformation of dsDNA is believed to be truly $2 \mathrm{D}$ equilibrated, ${ }^{16}$ while projected $3 \mathrm{D}$ structures have been found in other studies. ${ }^{17}$ As we find a critical exponent close to the $2 \mathrm{D}$ value we interpret the conformation of the ssDNA molecules as being 2D. Another issue is the change of the persistence length with salt concentration. In solution many different studies have addressed this question, see Ref. 5 and references therein. For molecules adsorbed on a surface the situation is different, and the relevant expression is changed. ${ }^{15}$

If we compare our finding regarding the persistence length to the literature we observe a reasonably good agreement: Using fluorescence recovery after photobleaching Tinland et al. ${ }^{14}$ found $\ell_{p}$ to be, depending on hydrodynamic model, 0.8 or $1.3 \mathrm{~nm}, 2.6$ or $4.4 \mathrm{~nm}$, and 4.9 or $8.4 \mathrm{~nm}$ in 100,10 , and $1 \mathrm{mM}$ salt solutions, respectively. These results are in very good agreement with ours and the data point for $1 \mathrm{mM} \mathrm{NaCl}$ overlap perfectly. However, Tinland et al. estimated the persistence length using the relation $R_{G}^{2} \propto \ell_{p} L$, and besides that, the molecules have been labeled by YOYO. Mechanical stretching of ssDNA gave $\ell_{p}$ around $0.8 \mathrm{~nm}$ (Ref. 13) in a $150 \mathrm{mM} \mathrm{NaCl}$ solution. As mentioned by the authors secondary structure may have influenced the results. To distinguish the different contributions to ssDNA stiffness from backbone flexibility and base stacking, synthetic sequences have also been studied. Fluorescence spectroscopy on poly $(\mathrm{dT})$ yielded between $1.5 \mathrm{~nm}(2 \mathrm{M} \mathrm{NaCl})$ and $3 \mathrm{~nm}$ $(25 \mathrm{mM} \mathrm{NaCl}) .^{7}$ Studies of poly $(\mathrm{dA})$ and poly $(\mathrm{dT})$ using double-stranded sequences with single-stranded gaps ${ }^{6}$ emphasized the importance of stacking interactions, giving $\ell_{p}$ $\simeq 2.5 \mathrm{~nm}$ for $\operatorname{poly}(\mathrm{dT})$ and $\ell_{p} \simeq 7.8 \mathrm{~nm}$ for poly $(\mathrm{dA})$, the latter having more pronounced stacking interactions. There has, however, been some debate regarding the difference in stiffness for poly $(\mathrm{dA})$ and poly $(\mathrm{dT}) .^{8-10}$ Recently, a study found values of $\ell_{p}$ of $1.13 \mathrm{~nm}$ for poly(A) and $1.71 \mathrm{~nm}$ for poly $(\mathrm{C})$ based on force-extension data at $500 \mathrm{mM} \mathrm{NaCl} .{ }^{11} \mathrm{In}$ a related study the same group found $\ell_{p} \simeq 1 \mathrm{~nm}$ for poly $(\mathrm{U}) .^{12}$

\section{CONCLUSION}

We have studied the stiffness and scaling properties of circular ssDNA. The stiffness was quantified using the persistence length, which we obtained from three quantities: (1) the exponential decay of the bond-correlation function for nearby segments, (2) the WLC expression for the end-to-end distance, and (3) the distribution function of end-to-end distances for a semiflexible chain. At a very low ionic strength of the solution, we found a value of $\ell_{p}$ around $9 \mathrm{~nm}$ from the first two quantities, while the distribution function gave a slightly lower value. Increasing the salt concentration we found a clear decrease in the persistence length, to about 6 $\mathrm{nm}$ at $1 \mathrm{mM} \mathrm{NaCl}$, and $4.5 \mathrm{~nm}$ at $10 \mathrm{mM} \mathrm{NaCl}$. The values were obtained from purely single-stranded regions of the molecules. We found a scaling behavior consistent with a 2D SAW, i.e., a scaling exponent of 0.75 for the end-to-end distance. The $2 \mathrm{D}$ behavior was further confirmed by analyzing the end-to-end distance distribution for large separations along the contour.

\section{ACKNOWLEDGMENTS}

We thank P. De Los Rios, S. Levene, A. Stasiak, and K. Yoshikawa for discussions, and B. Duplantier, E. Frey, and R. Metzler for discussions and interpretation of the data. G.D. thanks the Swiss National Science Foundation for support through Grant No. 200021-116515. K.R. acknowledges the Carlsberg and Novartis Foundations for financial support.

${ }^{1}$ M. E. Hogan and R. H. Austin, Nature (London) 329, 263 (1987).

${ }^{2}$ A. Montanari and M. Mézard, Phys. Rev. Lett. 86, 2178 (2001).

${ }^{3}$ M.-N. Dessinges, B. Maier, Y. Zhang, M. Peliti, D. Bensimon, and V. Croquette, Phys. Rev. Lett. 89, 248102 (2002).

${ }^{4}$ M. Rubinstein and R. H. Colby, Polymer Physics (Oxford University Press, Oxford, 2003).

${ }^{5}$ A. V. Dobrynin, Macromolecules 38, 9304 (2005).

${ }^{6}$ J. B. Mills, E. Vacano, and P. J. Hagerman, J. Mol. Biol. 285, 245 (1999).

${ }^{7}$ M. C. Murphy, I. Rasnik, W. Cheng, T. M. Lohman, and T. Ha, Biophys. J. 86, 2530 (2004)

${ }^{8}$ N. L. Goddard, G. Bonnet, O. Krichevsky, and A. Libchaber, Phys. Rev. Lett. 85, 2400 (2000).

${ }^{9}$ A. Ansari, Y. Shen, and S. V. Kuznetsov, Phys. Rev. Lett. 88, 069801 (2002).

${ }^{10}$ N. L. Goddard, G. Bonnet, O. Krichevsky, and A. Libchaber, Phys. Rev. Lett. 88, 069802 (2002).

${ }^{11}$ Y. Seol, G. M. Skinner, K. Visscher, A. Buhot, and A. Halperin, Phys. Rev. Lett. 98, 158103 (2007).

${ }^{12}$ Y. Seol, G. M. Skinner, and K. Visscher, Phys. Rev. Lett. 93, 118102 (2004).

${ }^{13}$ S. B. Smith, Y. Cui, and C. Bustamante, Science 271, 795 (1996).

${ }^{14}$ B. Tinland, A. Pluen, J. Sturm, and G. Weill, Macromolecules 30, 5763 (1997).

${ }^{15}$ R. R. Netz and J.-F. Joanny, Macromolecules 32, 9013 (1999).

${ }^{16}$ C. Rivetti, M. Guthold, and C. Bustamante, J. Mol. Biol. 264, 919 (1996).

${ }^{17}$ F. Valle, M. Favre, P. De Los Rios, A. Rosa, and G. Dietler, Phys. Rev. 
Lett. 95, 158105 (2005).

${ }^{18}$ N. Yoshinaga, K. Yoshikawa, and S. Kidoaki, J. Chem. Phys. 116, 9926 (2002).

${ }^{19}$ A. Podestà, M. Indrieri, D. Brogioli, G. S. Manning, P. Milani, R. Guerra, L. Finzi, and D. Dunlap, Biophys. J. 89, 2558 (2005).

${ }^{20}$ M. L. Sushko, A. L. Shluger, and C. Rivetti, Langmuir 22, 7678 (2006).

${ }^{21}$ B. Maier and J. O. Rädler, Phys. Rev. Lett. 82, 1911 (1999).

${ }^{22}$ B. Maier and J. O. Rädler, Macromolecules 33, 7185 (2000).

${ }^{23}$ T. Thundat, D. P. Allison, R. J. Warmack, M. J. Doktycz, K. B. Jacobson, and G. M. Brown, J. Vac. Sci. Technol. A 11, 824 (1993).

${ }^{24}$ T. Matsumoto, Y. Meada, Y. Naitoh, and T. Kawai, J. Vac. Sci. Technol. B 17, 1941 (1999).

${ }^{25}$ A. T. Woolley and R. T. Kelly, Nano Lett. 1, 345 (2001).

${ }^{26}$ J. Adamcik, D. V. Klinov, G. Witz, S. K. Sekatskii, and G. Dietler, FEBS Lett. 580, 5671 (2006).

${ }^{27}$ D. Klinov, B. Dwir, E. Kapon, N. Borovok, T. Molotsky, and A. Kotlyar, Nanotechnology 18, 225102 (2007).

${ }^{28}$ http://www.nanotuning.com.

${ }^{29}$ Y. Lyubchenko, L. Shlyakhtenko, R. Harrington, P. Oden, and S. Lindsay, Proc. Natl. Acad. Sci. U.S.A. 90, 2137 (1993).

${ }^{30}$ M. Bezanilla, S. Manne, D. E. Laney, Y. L. Lyubchenko, and H. G. Hansma, Langmuir 11, 655 (1995).

${ }^{31}$ J. Marek, E. Demjénová, Z. Tomori, J. Janáček, I. Zolotová, F. Valle, M. Favre, and G. Dietler, Cytometry 63A, 87 (2005).

${ }^{32}$ C. Domb and F. T. Hioe, J. Chem. Phys. 51, 1920 (1969).

${ }^{33}$ G. Witz, K. Rechendorff, J. Adamcik, and G. Dietler, Phys. Rev. Lett. 101, 148103 (2008).

${ }^{34}$ A. Baumgärtner, J. Chem. Phys. 76, 4275 (1982).

${ }^{35}$ The exponential decrease is given as $\exp \left(-s / \ell_{p}^{D}\right)$ in any dimensionality $D$. The value found for $\ell_{p}^{D}$ from this formula is then the one pertaining to $D$-dimensional space into which the polymer is embedded. For a WLC model there exist a relation between the 3D and 2D one: $\ell_{p}^{2 D}=2 \ell_{p}^{3 D}$. Thus, using the $2 \ell_{p}$ as decay constant allows us to find the relevant persistence length for $3 \mathrm{D}$, assuming that the surface introduces no additional complications during the adsorption.

${ }^{36}$ The bond correlations and the end-to-end distance are related: $\left\langle\xi^{2}\right\rangle$ $=\sum_{i}\left\langle\vec{u}_{i} \cdot \vec{u}_{i}\right\rangle+\sum \sum_{i \neq j}\left\langle\vec{u}_{i} \cdot \vec{u}_{j}\right\rangle$, where $\vec{u}_{i}$ describes the $i$ th bond-vector. The direct term leads to the usual Gaussian scaling in the absence of correlations among different segments $\left\langle\vec{u}_{i} \cdot \vec{u}_{j}\right\rangle=0$; however, it is clear that in order to get a different scaling exponent, the second correlation term must be dominant in the limit of large chains. Hence, if one observes SAW scaling in the end-to-end distance, the bond correlations must necessarily deviate from a simple exponential form, see Ref. 32.

${ }^{37}$ V. Bloomfield and B. H. Zimm, J. Chem. Phys. 44, 315 (1966).

${ }^{38}$ G. Witz, T. Eggel, P. De Los Rios, J. Adamcik, K. Karczewski, and G. Dietler (unpublished).

${ }^{39}$ C. J. Camacho, M. E. Fisher, and R. R. P. Singh, J. Chem. Phys. 94, 5693 (1991).

${ }^{40}$ J. Wilhelm and E. Frey, Phys. Rev. Lett. 77, 2581 (1996).

${ }^{41}$ R. G. Winkler, J. Chem. Phys. 118, 2919 (2003).

${ }^{42}$ J. K. Bhattacharjee, D. Thirumalai, and J. D. Bryngelson, arXiv:condmat/9709345.

${ }^{43}$ B. Hamprecht and H. Kleinert, Phys. Rev. E 71, 031803 (2005).

${ }^{44}$ R. Zandi, J. Rudnick, and R. Golestanian, Phys. Rev. E 67, 021803 (2003).

${ }^{45}$ The formula for $f(\xi)$ is given as a sum over parabolic cylinder functions multiplied by an exponential. Due to the rapid convergence we use only the first term, $\ell=0$ in the terminology of Ref. 40 .

${ }^{46}$ M. E. Fisher, J. Chem. Phys. 44, 616 (1966).

${ }^{47}$ M. Wittkop, S. Kreitmeier, and D. Göritz, J. Chem. Phys. 104, 351 (1996).

${ }^{48}$ B. Duplantier, Phys. Rev. B 35, 5290 (1987). 